\title{
The Epidemic Volatility Index: an early warning tool for epidemics
}

Polychronis Kostoulas $^{1}$, Eletherios Meletis ${ }^{1}$, Konstantinos Pateras ${ }^{1}$, Paolo Eusebi ${ }^{2}$, Theodoros Kostoulas ${ }^{3}$, Niko Speybroeck ${ }^{4}$, Matthew Denwood ${ }^{5}$, Suhail A.R. Doi ${ }^{6}$, Christian L. Althaus ${ }^{7}$, Carsten Kirkeby ${ }^{5}$, Pejman Rohani ${ }^{8}$, Navneet K. Dhand ${ }^{9}$, José L. Peñalvo ${ }^{10}$, Luis Furuya-Kanamori ${ }^{11}$, Lehana Thabane ${ }^{12}$, Slimane BenMiled ${ }^{13}$, Hamid Sharifi ${ }^{14}$, and Stephen Walter ${ }^{12}$

${ }^{1}$ Faculty of Public Health, University of Thessaly, Greece.

${ }^{2}$ Department of Medicine and Surgery, University of Perugia.

${ }^{3}$ Department of Information and Communication Systems Engineering, University of the Aegean, Greece

${ }^{4}$ Research Institute of Health and Society (IRSS), Université Catholique de Louvain, 1200, Brussels, Belgium.

${ }^{5}$ Department of Veterinary and Animal Sciences, University of Copenhagen, Denmark

${ }^{6}$ Department of Population Medicine, College of Medicine, QU Health, Qatar University, Doha, Qatar.

${ }^{7}$ Institute of Social and Preventive Medicine, University of Bern, Bern, Switzerland.

${ }^{8}$ Center for the Ecology of Infectious Diseases, University of Georgia, Athens, GA 30602, USA.

${ }^{9}$ Sydney School of Veterinary Science, The University of Sydney, Camden, NSW, Australia.

${ }^{10}$ Unit of Noncommunicable Diseases, Department of Public Health, Institute of Tropical Medicine, Antwerp, Belgium.

${ }^{11}$ UQ Centre for Clinical Research, Faculty of Medicine, The University of Queensland, Herston, Australia.

${ }^{12}$ Department of Health Research Methods, Evidence, and Impact, McMaster University, Hamilton ON, Canada.

${ }^{13}$ Pasteur Institute, University of Tunis el Manar, Tunis, Tunisia.

${ }^{14} \mathrm{HIV} / \mathrm{STI}$ Surveillance Research Center, and WHO Collaborating Center for HIV Surveillance, Institute for Futures Studies in Health, Kerman University of Medical Sciences, Kerman, Iran.

May 29, 2021

\footnotetext{
Abstract

Background. This paper presents, for the first time, the Epidemic Volatility Index (EVI), a conceptually simple, early warning tool for emerging epidemic waves.

Methods. EVI is based on the volatility of the newly reported cases per unit of time, ideally per day, and issues an early warning when the rate of the volatility change exceeds a threshold.

Results. Results from the COVID-19 epidemic in Italy and New York are presented here, while daily updated predictions for all world countries and each of the United States are available online.
} 
Interpretation. EVI's application to data from the current COVID-19 pandemic revealed a consistent and stable performance in terms of detecting oncoming waves. The application of EVI to other epidemics and syndromic surveillance tasks in combination with existing early warning systems will enhance our ability to act fast and optimize containment of outbreaks.

\section{Introduction}

Early warning tools are crucial for the timely application of intervention strategies and the mitigation of the adverse health, social and economic effects associated with epidemics. Sentinel networks in combination with information technology infrastructures in public health ${ }^{1}$ provide data for the detection of spatial and temporal aberrations in the expected number of cases for groups of clinical signs and symptoms ${ }^{2}$. Several modelling frameworks exist for the analysis of such data. For example, the moving epidemic method, an approach used to monitor, among others, the start of the flu epidemic ${ }^{3}$. Further, methods based on seasonality patterns, the link between pathogens and meteorological parameters ${ }^{4}$ and/or the measurement of vector indices for vector-borne pathogens ${ }^{5}$ are also available.

Once an epidemic erupts, growth models can be used to predict the course of the outbreak and quantify its consequences. The advantages and limitations of these methods have been extensively discussed ${ }^{6}$. Machine learning algorithms have also been utilized with the most recent application being in the current COVID-19 pandemic ${ }^{7}$. Correlating the number of COVID-19 cases with parameters obtained using big data approaches can predict future rise in the number of cases. For example, monitoring of digital data streams can provide an early indication of a rise in the COVID-19 cases and deaths in the next 2 to 3 weeks ${ }^{8}$. All models have limitations arising from the imperfect nature of the data. The need for open, better, detailed data is imperative for the deployment of models with improved accuracy, models that will have better predictive ability and will be more useful for the timely application of appropriate control measures for the COVID-19 pandemic ${ }^{9}$.

Our work introduces the Epidemic Volatility Index (EVI), which is inspired by the use of volatility indices in the stock market ${ }^{10,11}$. EVI is based on the moving standard deviation of the newly reported cases during an epidemic. First we present the rationale of EVI and then provide an example application with COVID19 data from Italy and New York. Daily updated predictions - with a 48 hour lag for confirmation purposes - are available online for all world countries and each of the United States. Results revealed a firm and consistent ability of EVI to predict the COVID-19 epidemic waves, in all instances.

\section{Methods}

\section{The Epidemic Volatility Index}

EVI is calculated for a rolling window of time series epidemic data (i.e. the number of new cases per day). At each step, the observations within the window are obtained by shifting the window forward over the time series data one observation at a time.

Let $x_{i}=\left\{x_{1}, x_{2}, \ldots, x_{n}\right\}$ be a time series of length $N$. The rolling window size - that is the number of consecutive observations per rolling window - is $m$. With $0<m \leq m_{\max }$ and $0<m_{\max } \leq N$, there are $t=N-m+1$ consecutive rolling windows. 
At each of the $t$ steps, EVI uses the standard deviation $\left(s_{t}\right)$ of the newly reported cases $\left(y_{j_{t}}=\left\{y_{1_{t}}, y_{2_{t}}, \ldots, y_{m_{t}}\right\}\right)$ within the specified $m$

$$
s_{t}=\sqrt{\frac{1}{m} \sum_{i_{t}=1}^{m}\left(x_{i_{t}}-\bar{x}_{t}\right)^{2}}
$$

with $\overline{x_{t}}$ the mean of the $t^{t h}$ window. Subsequently, EVI is calculated as the relative change of $\left(s_{t}\right)$ between two consecutive rolling windows:

$$
E V I_{t-1, t}=\frac{s_{t}-s_{t-1}}{s_{t}}
$$

We expect an increase in the future number of cases, if $E V I_{t-1, t}$ exceeds a threshold $c(c \in[0,1])$ and the observed cases at time point $t,\left(y_{t}\right)$ are higher than the average of the reported cases in the previous week:

$$
\operatorname{Ind}_{E V I_{t-1, t}}= \begin{cases}1 & \text { if } E V I_{t-1, t} \geq c \wedge y_{t} \geq \bar{\mu}_{t: t-7} \\ 0 & \text { otherwise }\end{cases}
$$

\section{Case definition and desired accuracy}

The user should provide the minimum rise in cases that, if present, should be detected. A case definition can be the rise in mean the number of cases between two consecutive weeks that exceeds a threshold $r$ :

$$
\frac{\bar{\mu}_{t: t-7}}{\bar{\mu}_{t: t+7}} \leq r
$$

with $0 \leq r \leq 1$.

The accuracy of EVI, given the specified case definition, depends on $m$ and $c$, which should be selected in a way to achieve a desired accuracy target. Several strategies are available. One option is the selection of $m$ and $c$ values that lead to the simultaneous optimization of the sensitivity $(S e)$ and the specificity $(S p)$ for EVI, the maximization of the Youden index $(J=S e+S p-1)^{12}$ and, hence, the overall minimization of the false results (i.e. both false positive and false negative early warnings). Another approach could be to select $m$ and $c$ such that the highest $S e($ or $S p)$ is achieved with $S p($ or $S e)=1$ or not dropping below a critical value (e.g. 95\%). Advanced Receiver Operating Characteristic curve analysis can also be performed ${ }^{13}$ and selection of critical values can be based on indices that quantify the relative cost of false positive (i.e., falsely predicting an upcoming epidemic wave) to false negative (i.e., failing to predict an upcoming epidemic wave) warnings, like the misclassification cost term $(M C T)$.

\section{Generation of an early warning}

Every time a new time point $t$ is observed, the model uses all of the observed cases up to $t$ to decide whether it should issue an early warning, at time point $t$. The steps are:

1. Observed cases up to $t$ are analyzed for all possible values of the window size $\left(m \in\left[1, m_{\max }\right]\right)$ and threshold $(c \in[0,1])$.

2. For each of the $m$ and $c$ combinations, the $S e_{t_{m, c}}$ and $S p_{t_{m, c}}$ are estimated for the predefined case definition (Eq. 4). 
3. The $m^{\prime}$ and $c^{\prime}$ that give the best $S e_{t_{m^{\prime}, c^{\prime}}}$ and $S p_{t_{m^{\prime} c^{\prime}}}$ combination are selected.

4. For $m^{\prime}$ and $c^{\prime}$, the value of $I n d_{E V I t, t-1}$ is determined at the most recent time point $t$ and a decision is made on whether or not a warning signal is issued.

\section{Accuracy and Predictive Values}

Further, at each time point $t$, the probability of observing a rise or drop in the future cases, given that an early warning was issued or not, can be calculated as the positive $\left(P V_{t}+\right)$ and negative $\left(P V_{t}-\right)$ predictive value, respectively:

$$
\begin{gathered}
P V_{t}+=P(D+\mid T+)=\frac{p_{1: t} S e_{t_{m^{\prime}, c^{\prime}}}}{p_{1: t} S e_{t_{m^{\prime}, c^{\prime}}}+\left(1-p_{1: t}\right)\left(1-S p_{t_{m^{\prime}, c^{\prime}}}\right)} \\
P V_{t}-=P(D-\mid T-)=\frac{\left(1-p_{1: t}\right) S p_{t_{m^{\prime}, c^{\prime}}}}{\left(1-p_{1: t}\right) S p_{t_{m^{\prime}, c^{\prime}}}+p_{1: t}\left(1-S e_{t_{m^{\prime}, c^{\prime}}}\right)}
\end{gathered}
$$

where $p_{1: t}$ is the proportion of events satisfying the condition of Eq. 4 up to time point $t$.

Once the entire time series data have been observed, the overall $S e_{E V I}$ can be estimated as the fraction of the total number of occurrences for which an early warning has been issued, given that the case definition (Eq. 4 ) holds $(P(T+\mid D+))$, divided by the total number of occurrences that the case definition holds $(P(D+))$. Similarly, the overall $S p_{E V I}$ is calculated as the fraction of the total number of occurrences for which an early warning was not issued given that the expected rise of cases was not observed, that is, the case definition is not true, $(P(T-\mid D-))$ divided by the total number of occurrences that the case definition is not true $(P(D-))$ :

$$
S e_{E V I}=\frac{P(T+\mid D+)}{P(D+)}, S p_{E V I}=\frac{P(T-\mid D-)}{P(D-)}
$$

\section{Sensitivity analysis}

The performance of EVI depends on the specified case definition (i.e., $r$ ) and the desired accuracy. Ideally, in the presence of historical data, various case definitions and $r$ values should be explored to identify combinations that provide the optimal monitoring of an epidemic.

\section{Example application}

The current most serious threat to global health and economy ${ }^{14}$ is the COVID-19 pandemic that begun in China and was first reported to the WHO China Country Office on December 31, $2019^{15}$. Data on the confirmed cases of COVID-19 were retrieved from the COVID-19 Data Repository, which is maintained by the Center for Systems Science and Engineering (CSSE) at Johns Hopkins University ${ }^{16}$. The number of daily confirmed new cases of COVID-19, for each country, from January 22, 2020 until April 13, 2021 were analyzed. Due to unnatural variability in the reported cases between working days and weekends, the 7-day moving average rather than the actual observed cases were analyzed. For the analysis, $m_{\max }$ was restricted to 30 days in order to avoid the effect of potentially higher volatility from previous epidemic waves on the volatility estimates of the most recent data and the predictive ability of EVI for upcoming and perhaps milder epidemic waves. 
The case definition was an increase in the mean of expected cases, between two consecutive weeks, equal or higher than twenty percent, $r \leq \frac{1}{1.2}$. For sensitivity analysis, the detection of an increase in the mean of expected cases equal or higher than 50 percent $\left(r \leq \frac{1}{1.5}\right)$ was considered. Data were analyzed separately for each country and for each of the states of the United States of America that had experienced a total number of cases higher than 20,000, until April 13, 2021.

\section{Statistical software}

All models were run in $\mathrm{R}^{17}$. The packages readxl ${ }^{18}, \operatorname{ggplot} 2^{19}$, $\operatorname{cowplot}^{20}$ and $\operatorname{readr}{ }^{21}$ were used.

\section{Results}

Results for Italy, one of the most severely affected EU countries ${ }^{22}$, and New York, which was in the epicenter of the pandemic in the U.S. ${ }^{23}$, are presented in the main manuscript. Daily updated results for all world coutnries and each of the United States are available online at http://83.212.174.99:3838.

Confirmed COVID-19 cases for Italy and New York State, from January 22, 2020, until April 13, 2021, are in Figures 1 and 2, respectively. Red dots correspond to time points that an early warning was issued according to $I n d_{E V I_{t, t-1}}$, while grey dots to time points without an early warning indication. Further, the positive and negative predictive values at each time point are in Figures 3 and 4, respectively.
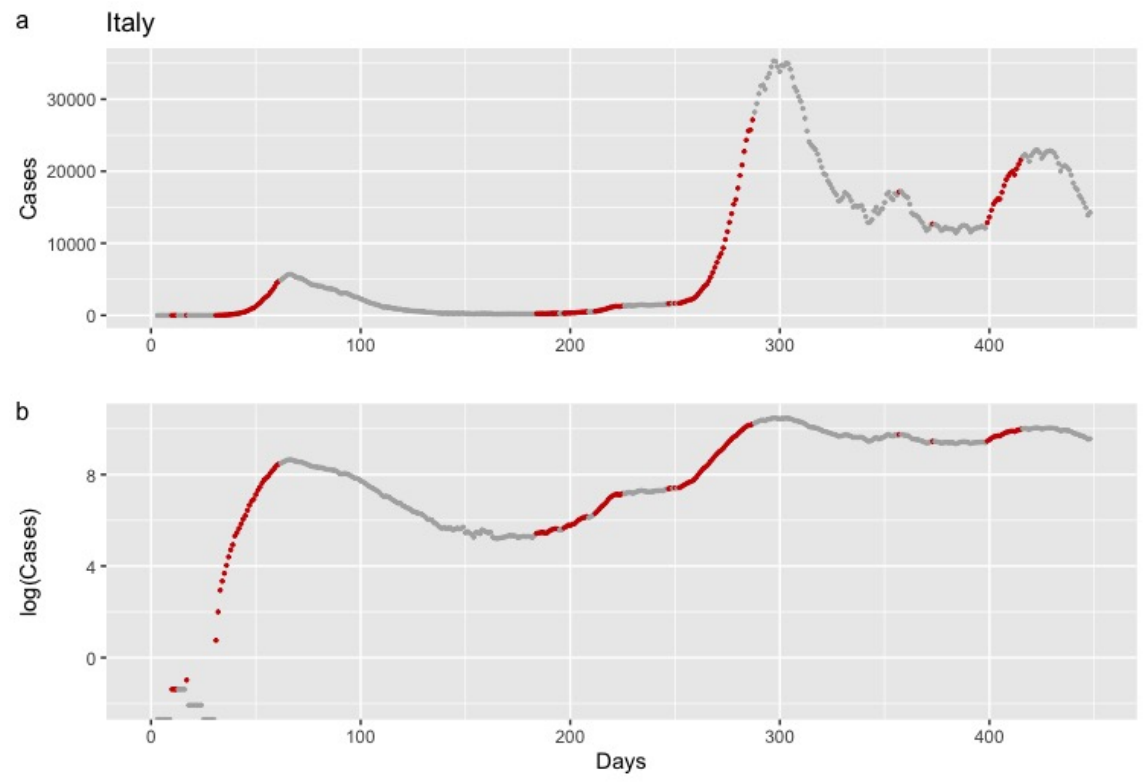

Figure 1: Daily confirmed cases of COVID-19 in Italy, from January 22, 2020 until April 13, 2021. Red dots correspond to dates that, according to the Epidemic Volatility Index (EVI), an early warning was issued indicating that a rise in the COVID-19 cases is expected. Data are presented on the original scale (1a) and the logarithmic scale (1b), which facilitates the comparison of the steepness of the epidemic curve between the different waves. 

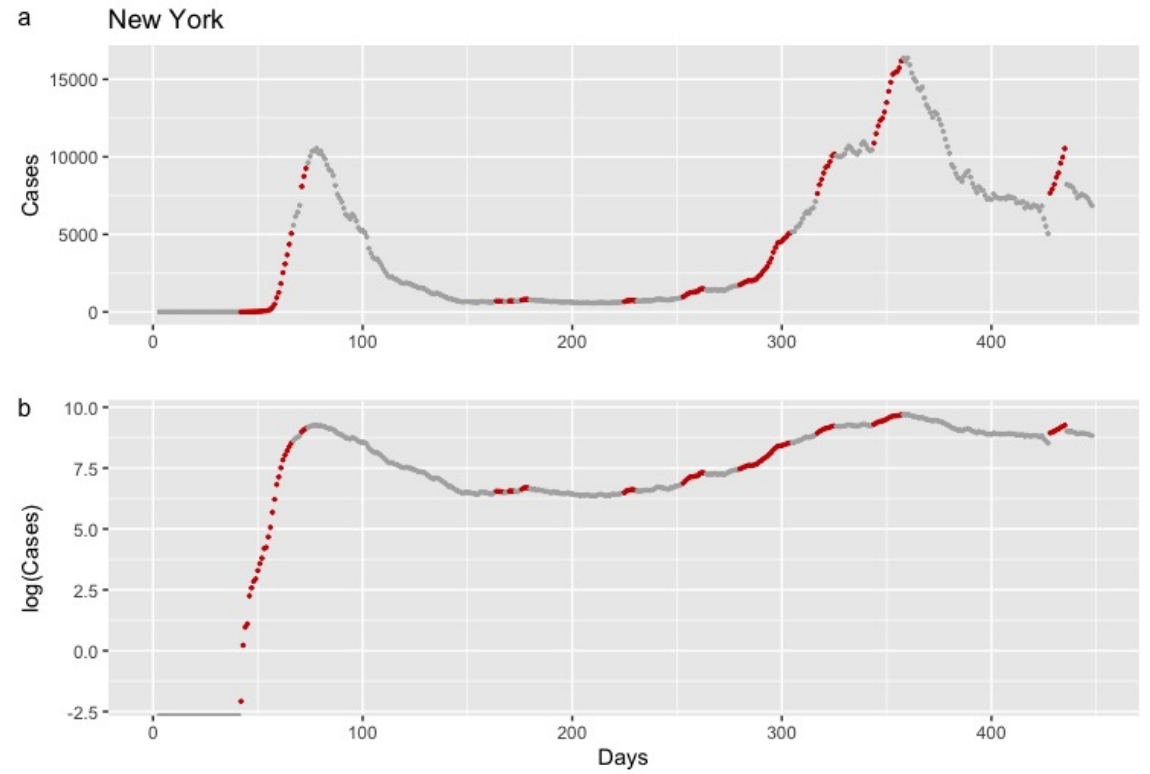

Figure 2: Daily confirmed cases of COVID-19 in New York, from January 22, 2020 until April 13, 2021. Red dots correspond to dates that, according to EVI, an early warning was issued indicating that a rise in the COVID-19 cases is expected. Data are presented on the original scale (1a) and the logarithmic scale (1b), which facilitates the comparison of the steepness of the epidemic curve between the different waves.
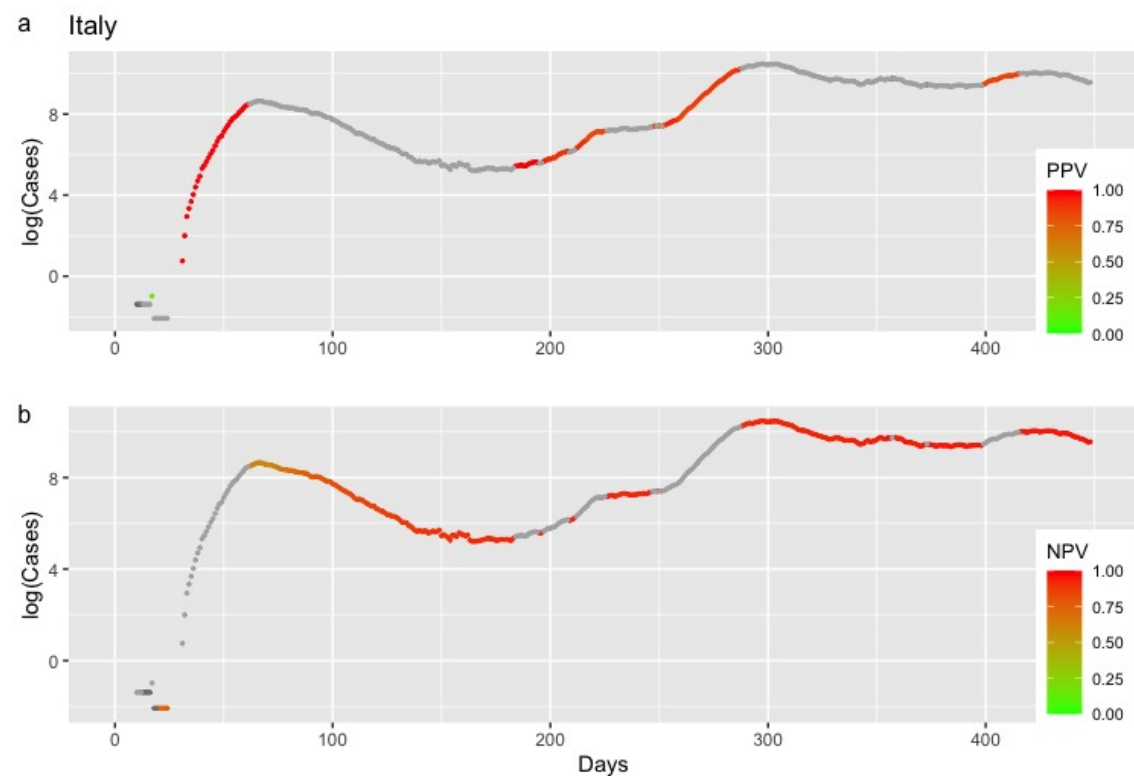

Figure 3: Positive and negative predictive values (PPV in 3a and NPV in 3b), for Italy, depending on whether or not an early warning was issued. Higher color intensity corresponds to predictive values closer to the value of 1 . 

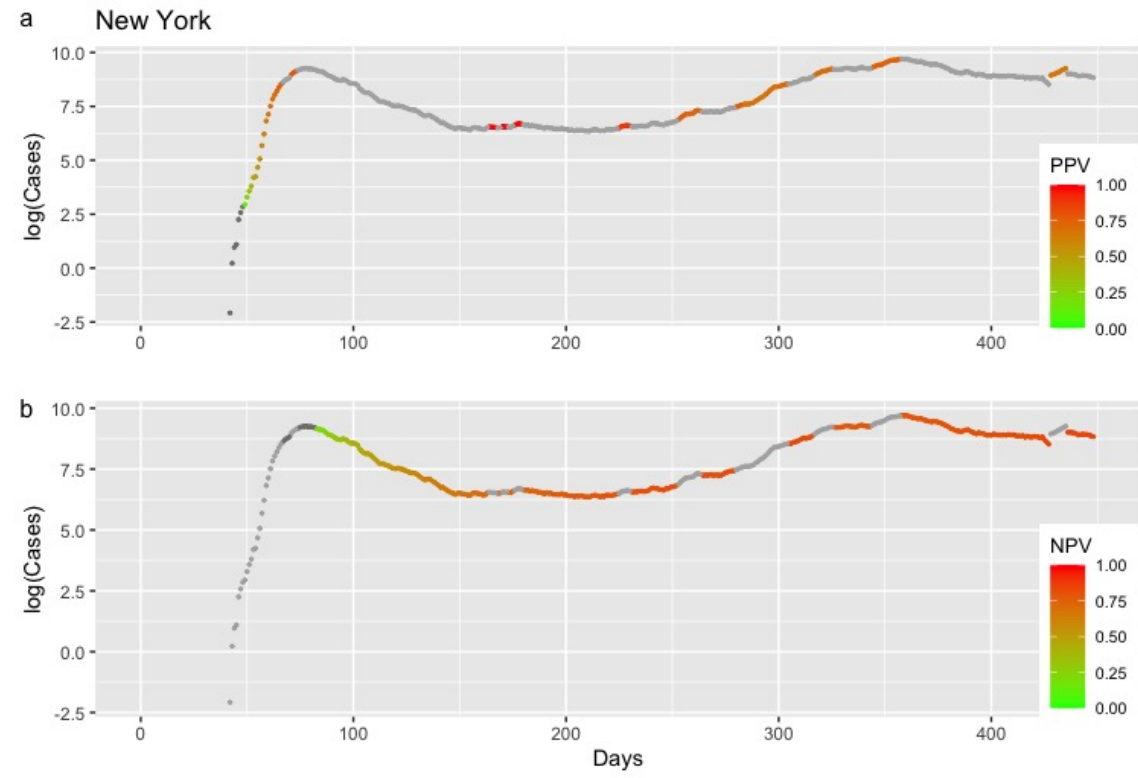

Figure 4: Positive and negative predictive values (PPV in 4a and NPV in 4b), for New York, depending on whether or not an early warning was issued. Higher color intensity corresponds to predictive values closer to the value of 1 .

For Italy, the overall sensitivity for EVI was $0.82(0.75 ; 0.89)$ and the specificity was $0.91(0.88 ; 0.94)$. For New York, the corresponding values were $0.55(0.47 ; 0.64)$ and $0.88(0.84 ; 0.91)$.

Sensitivity analysis results for Italy, under alternative $r$ specification (i.e., $r=\frac{1}{1.5}$ ), are in Figure 5 . The overall sensitivity and specificity, for $r=\frac{1}{1.5}$, were $0.75(0.66 ; 0.85)$ and $0.93(0.91 ; 0.96)$, respectively. 

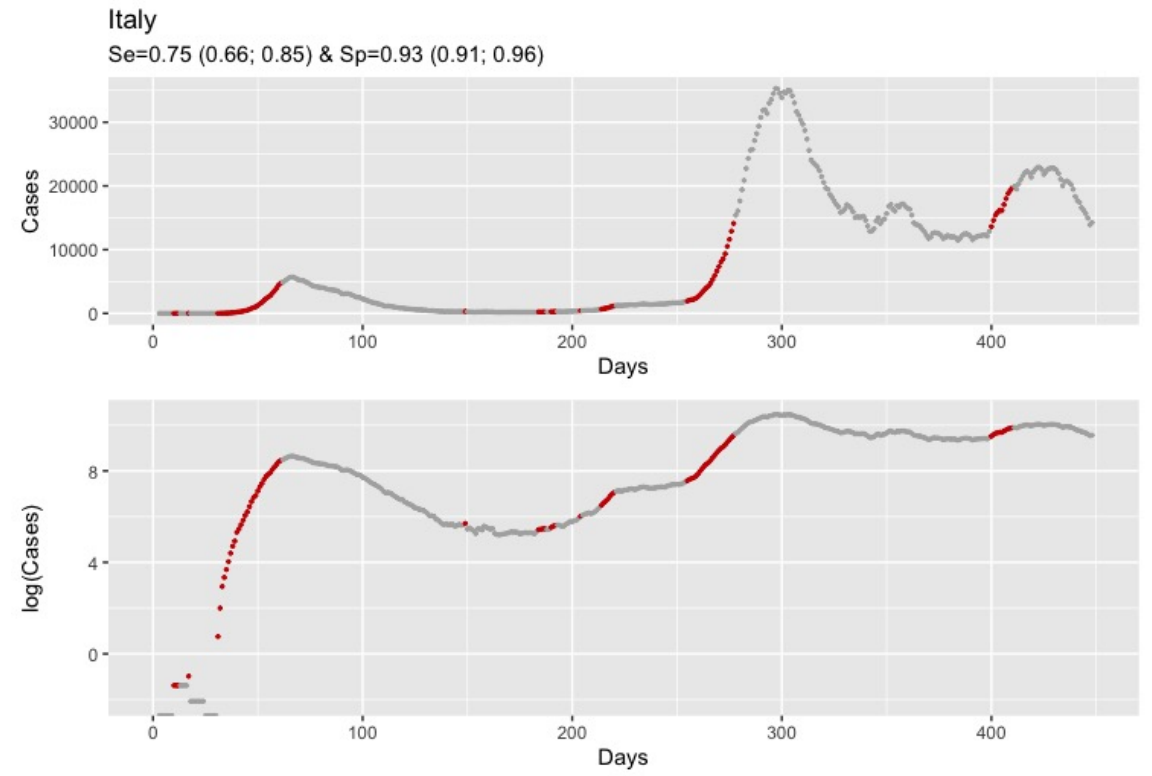

Figure 5: Daily confirmed cases of COVID-19 in Italy, from January 22, 2020 until April 13, 2021. Analysis with $r=\frac{1}{1.5}$. Red dots correspond to dates that, based on EVI, an early warning was issued indicating that a rise in the COVID-19 cases is expected. Data are presented on the original scale (1a) and the logarithmic scale (1b) which facilitates the comparison of the steepness of the epidemic curve between the different waves.

A common and consistent finding in the results from all countries was that repetitive early warnings are linked to the start of a new epidemic wave, while the absence of warnings indicates a stable course or a future drop in the number of new COVID-19 cases (Fig. 1, 2 and http://83.212.174.99:3838/).

\section{Discussion}

EVI is an efficient and easy to implement early-warning tool for an upcoming rise in the number of new cases. The performance of EVI, as expressed by its overall $S e$ and $S p$, was, in all instances, high. A more important aspect lies in the fact that repetitive issuance of early warnings indicates the beginning of an epidemic wave. This is a consistent and remarkably stable finding across all countries and each of the United States (Fig. 1, 2 and http://83.212.174.99:3838/). In a similar manner, the absence of a series of early warnings implies that the number of new cases will remain stable or drop. The latter was also a consistent finding. Additionally, false early warnings (i.e. false positives) were isolated instances and did not occur in a consecutive series. There were few occasions with a consecutive absence of early warnings despite a continuing rise in the number of cases (i.e. false negatives). Nevertheless, such series of false negatives were always close to the peak of a wave. This finding is reasonable and could be interpreted as an early sign of reaching the peak because EVI depends on volatility and the increase in the number of new cases decelerates when approaching the peak of an epidemic wave. Positive and negative predictive values that are calculated at each time point can also be used to assess the probability that an early warning, or its absence, is true. In all instances, predictive values were high with the exception of few instances at the beginning of the time series due to the absence of enough data.

Work on the SIR and SIS models has revealed that moving-window estimates of the variance increase while approaching the emergence of a pathogen as well as during the elimination phase and that it can be used as an early warning tool ${ }^{24}$. EVI is based on the relative rather than the absolute change of the standard 
deviation because the latter depends on the underlying prevalence at each time point of the epidemic. Hence, a low threshold would be efficient in detecting a surge in the new cases at the beginning of an epidemic, when the baseline prevalence is low, but would have failed to do so for subsequent epidemic waves that commence from a higher baseline prevalence. On the other hand, a high absolute threshold would have failed to capture waves at the beginning of the epidemic. EVI is based on the relative increase in volatility, which implicitly adjusts for the baseline prevalence at each point of the time series.

In general, the ability of EVI to provide valid predictions does not seem to be affected by the fact that sampling and testing schemes for COVID-19 are mainly based on passive surveillance systems. EVI performed equally well among different countries with different control strategies, testing intensity and reporting accuracy and despite the fact that even within countries sampling and testing has changed over time and/or differs between regions ${ }^{25,26}$. Restriction of the maximum window size $\left(m_{\max }\right)$ to one month plays a key role, because reporting bias is expected to remain similar over short time periods. This form of non-differential misclassification leads to reporting rates that, though biased, do not have a significant impact on volatility, EVI and its predictive ability. Crucially, it is important that the data do not exhibit strong artifacts of recording bias, as there is no way for the method to distinguish between a trend due to underlying epidemic patterns and an observed trend due to changes in reporting practices or increased testing capacity or effort ${ }^{27}$. This could for instance happen when a country changes its general testing regime, experiences local outbreaks and focus testing in a specific area, or targets other subgroups of the population than previously. Thus, EVI should preferably be evaluated for use in smaller geographical regions, such as counties or municipalities, if sufficient, high quality data are available. Undoubtedly, all models are prone to limitations due to imperfect data ${ }^{9}$ but the continuing enhancement of active and passive surveillance systems - as the testing regimes and methods also improve - will lead to improved data quality.

The performance of EVI depends on the specified case definition and $r$, parameters which are epidemicspecific and country-specific. Modifications to allow for different case definition and $r$, for the different periods of an epidemic, are rather straightforward to implement. Parameters $c$ and $m$ are allowed to vary and take values that would satisfy the conditions set by the defined case and the desired accuracy. A point of concern is the selection of $m_{\max }$. For an ongoing epidemic with multiple waves, as is the case with COVID$19, m_{\max }$ should be limited to a period shorter than the entire observation period. This prevents excess volatility of past epidemic waves from affecting the most recent volatility estimates and the ability of EVI to warn for upcoming waves that may be smaller and of lower volatility than previous ones. In our example, we limited $m_{\max }$ to one month. EVI also depends on data intensity. Detailed data at the lowest time unit (i.e., days rather than weeks) is preferable in order to detect changes rapidly. In the COVID-19 example the 7-day moving average was analyzed instead of the daily reported cases because daily data had unnatural variability due to reporting variations between working days and weekends. Nevertheless, analysis based on the daily reported cases provided similar results (data not shown here).

Beyond the case of epidemics or rare events, like the COVID-19 pandemic, an important application of EVI can be in the context of syndromic surveillance ${ }^{28}$, not limited to outbreaks from terrorist attacks, but in its broader sense: the detection of temporal and spatial aberrations in the expected number of cases for signs and symptoms. Such systems already exist and utilize state-of-the-art information technologies within the context of public health ${ }^{1}$ as well as one health ${ }^{29,30}$. EVI can provide an additional early warning tool for these systems.

\section{References}

1Heffernan R, Mostashari F, Das D, Karpati A, Kulldorff M, Weiss D. Syndromic surveillance in public health practice, New York City. 2004.

2Brett TS, Rohani P. Dynamical footprints enable detection of disease emergence.. PLoS Biol 2020; 18: e3000697. 
3Vega T, Lozano JE, Meerhoff T, et al.. Influenza surveillance in Europe: establishing epidemic thresholds by the moving epidemic method. Influenza and other respiratory viruses 2013; 7: 546-58.

4Abeku TA, Hay SI, Ochola S, et al.. Malaria epidemic early warning and detection in African highlands. Trends in parasitology 2004; 20: 400-5.

5Chang F-S, Tseng Y-T, Hsu P-S, Chen C-D, Lian I-B, Chao D-Y. Re-assess vector indices threshold as an early warning tool for predicting dengue epidemic in a dengue non-endemic country. PLoS Negl Trop Dis 2015; 9: e0004043.

6Chowell G, Sattenspiel L, Bansal S, Viboud C. Mathematical models to characterize early epidemic growth: A review. Physics of life reviews 2016; 18: 66-97.

7Wang P, Zheng X, Li J, Zhu B. Prediction of epidemic trends in COVID-19 with logistic model and machine learning technics. Chaos, Solitons \& Fractals 2020; 139: 110058.

8Kogan NE, Clemente L, Liautaud P, et al.. An early warning approach to monitor COVID-19 activity with multiple digital traces in near real time.. Sci Adv 2021; 7.

9Vespignani A, Tian H, Dye C, et al.. Modelling COVID-19. Nature Reviews Physics 2020; 2: 279-81.

10Fernandes M, Medeiros MC, Scharth M. Modeling and predicting the CBOE market volatility index. Journal of Banking 63 Finance 2014; 40: 1-10.

11Brenner M, Galai D. New Financial Instruments for Hedge Changes in Volatility. Financial Analysts Journal 1989; 45: 61-5.

12Fluss R, Faraggi D, Reiser B. Estimation of the Youden Index and its Associated Cutoff Point. Biometrical Journal 2005; 47: 458-72.

13Zweig MH, Campbell G. Receiver-operating characteristic (ROC) plots: a fundamental evaluation tool in clinical medicine. Clinical Chemistry 1993; 39: 561-77.

14Fauci AS, Lane HC, Redfield RR. Covid-19 - Navigating the Uncharted. New England Journal of Medicine 2020; 382: 1268-9.

15Organization WH, others. Pneumonia of unknown cause - China: disease outbreak news. Geneva; January 5. 2020 .

16Dong E, Du H, Gardner L. An interactive web-based dashboard to track COVID-19 in real time.. Lancet Infect Dis 2020; 20: 533-4.

17R Core Team. R: A language and environment for statistical computing. 2020.

18Wickham H, Bryan J, Kalicinski M, et al.. Package 'readxl'. 2019.

19Wickham H. ggplot2. Wiley Interdisciplinary Reviews: Computational Statistics 2011; 3: 180-5.

20Wilke CO, Wickham H, Wilke MCO. Package 'cowplot'. Streamlined Plot Theme and Plot Annotations for 'ggplot2 2019.

21Wickham H, Hester J, Francois R, et al.. Package 'readr'. 2015.

22Livingston E, Bucher K. Coronavirus disease 2019 (COVID-19) in Italy. Jama 2020; 323: 1335-.

23Thompson CN, Baumgartner J, Pichardo C, et al.. COVID-19 Outbreak-New York City, February 29-June 1, 2020. Morbidity and Mortality Weekly Report 2020; 69: 1725.

24O'Regan SM, Drake JM. Theory of early warning signals of disease emergenceand leading indicators of elimination. Theoretical Ecology 2013; 6: 333-57. 
25Brynildsrud O. COVID-19 prevalence estimation by random sampling in population-optimal sample pooling under varying assumptions about true prevalence. BMC medical research methodology 2020; 20: 1-8.

26Middelburg RA, Rosendaal FR. COVID-19: How to make between-country comparisons. International Journal of Infectious Diseases 2020; 96: 477-81.

27Halasa T, Græsbøll K, Denwood M, Christensen LE, Kirkeby C. Prediction Models in Veterinary and Human Epidemiology: Our Experience With Modeling Sars-CoV-2 Spread. Frontiers in Veterinary Science 2020; 7. DOI:10.3389/fvets.2020.00513.

28Henning KJ. What is syndromic surveillance?. Morbidity and mortality weekly report 2004; : 7-11.

29Beltrán-Alcrudo D, Carpenter TE, Cardona C. A flock-tailored early warning system for low pathogenic avian influenza (LPAI) in commercial egg laying flocks.. Prev Vet Med 2009; 92: 324-32.

30Gilbert M, Golding N, Zhou H, et al.. Predicting the risk of avian influenza A H7N9 infection in livepoultry markets across Asia.. Nat Commun 2014; 5: 4116. 